\title{
DETERMINACIÓN DE LOS OBJETIVOS PARCIALES DE UN PROYECTO, APLICANDO LA RUTA MÁS LARGA
}

Determination of the Partial Objectives of a Project, Applying the Longest Route

Alicia preguntó al gato:

-¿Podrías decirme, por favor, qué camino he de tomar para salir de aquí?

- Depende mucho del punto adonde quieras ir- contestó el Gato.

-Me da casi igual dónde- dijo Alicia.

-Entonces no importa qué camino sigas- dijo el Gato".

Lewis Carroll

Universidad Politécnica de Guanajuato

Open Access bajo la licencia CC BY-NC-ND (http://creativecommons.org/licenses/by-nc-nd/4.0/).

Dr. Rafael Octavio Sainz Zamora

Profesor y asesor de la Maestría de

Comercialización de Conocimientos

Innovadores. UAEM
Inga. en Sist. Computacionales Ana Rosa Cornejo Martínez

L.A. Mayra Morán Castrejón

C.P. Rosa Araceli Torres Castañeda

Estudiantes de la Maestría de maestría en

Comercialización de Conocimientos

Innovadores.
Fecha de envío: 24 de abril 2016

Fecha de aceptación: 02 de julio 2016

Fecha de Publicación: 31 de Agosto 2016

$$
\text { No. de Reserva: 04-2014-081110025600-203 Vol.1, No.2 }
$$
2016 ISSN: 2007-977X 


\section{Resumen}

En el presente documento se aborda la problemática de lo que significa el establecer los objetivos parciales de un proyecto, cuando se minimiza su importancia o se carece de fundamento. En ocasiones se recurre al diagrama de Gantt como solución, pero como se verá más adelante, ésta no es la mejor opción. Un mal planteamiento de estos objetivos, puede alterar la asignación de tareas y por ello, el no arribar a los resultados esperados, en un descontrol manifiesto en recursos materiales y humanos.

La propuesta consiste en recurrir a la técnica de Project Evaluation and Review Technique, que permite determinar en tiempos estimados las actividades a desarrollar. De ahí, al agruparlas se conforman lo que son los objetivos parciales. Esta es una propuesta que evita la imposición de objetivos, ya que al aglutinar actividades de manera racional, estos objetivos se establecen como una consecuencia y no como una condición.

\section{Palabras clave; Objetivos parciales, Camino más largo, evaluación de proyectos, Gantt, metas.}

\section{Abstract}

This document is about the problem of what it means to establish the partial project objectives addressed when its importance is minimized or is unfounded. Sometimes they resort to Gantt chart as a solution, but as you will see later, this is not the best option. A wrong approach to these objectives can alter the allocation of tasks and therefore not arrive at the expected results, manifest lack of control in material and human resources.

The proposal is to use the technique of Project Evaluation and Review Technique, on estimates for determining the activities to time. Hence, to conform to group what are the partial objectives? This is a proposal that avoids imposing objectives, and activities that bring together rationally, these goals are established as a consequence and not as a condition.

\section{Key words: Partial Objectives, Critical Path, Project Evaluation, Gantt Chart, Goals.}

\section{Introducción}

Un proyecto de inversión es un plan prospectivo de una serie de acciones encaminadas a materializar algún objetivo, que bien puede ser del orden económico o social. A través de un proyecto de inversión, se definen y especifican las acciones conjuntas necesarias para dar solución a problemas o bien para cubrir las necesidades materiales.

Para Gómez, Coronel, Martínez y Llorente (2000) los proyectos de inversión siempre poseen una duración limitada, lo que implica el requerimiento de objetivos fijos que orienten hacia la efectividad de las acciones emprendidas. Por supuesto, que los objetivos del proyecto tienen como guía general la misión y visión de la empresa. La razón de los objetivos constituye la finalidad del proyecto y éstos deben responder a la pregunta: ¿Qué es lo que se pretende alcanzar con el proyecto?

\section{Los Objetivos}

En su ensayo "De Qué Hablo Cuando Hablo de Correr" Murakami cometa que él corre unos 10 kilómetros todos los días. Su objetivo no es correr cada vez más rápido, ni comparar su resultado con otros. En su carrera lo que valora es la búsqueda de su propio camino. Para, Koontz y Weihrich (1994) "La administración es el proceso de diseñar y mantener un entorno en el que, trabajando en grupos, los individuos cumplan eficientemente objetivos específicos", a lo que se agregan los propios y por supuesto los de la empresa. De una manera muy sencilla, el objetivo es, todos esos resultados que un negocio quiere alcanzar.

El objetivo, en nuestro caso, pone de relieve la estructura de las relaciones entre actividades cualitativas y cuantificables que caracteriza al proyecto y su contexto estratégico. Para Matus (1993) "El objetivo, es la definición del futuro pretendido y se deduce tanto de la misión, que delimita la razón de ser de la organización; como de la visión, que regula desde un plano holístico los valores de la organización. Es un lugar pragmático a conquistar por medio de la planificación y acción intencional". Los objetivos deben ser medibles y establecidos en responsabilidades definidas, con plazos de logro de manera sistemática para alcanzarlos. 
Churchman (1993) menciona que al resolver un problema, el proceso de razonamiento debe comenzar con el objetivo central del problema y luego los objetivos secundarios del mismo, los cuales son requeridos para lograr la atención del objetivo central de lo que se pretende resolver. Continuado con el pensamiento de Churchman, sustenta que el problema no sólo es la división entre causa y efecto, lo anterior se realiza por etapas, que son "actividades" que se realizan en un determinado tiempo. Estas etapas no siguen necesariamente una secuencia lineal a lo que el doctor llama "Razonamiento de etapas múltiples".

Robbins y De Cenzo (1994) sustentan que en el momento de estudiar un sistema, lo primero que hay que hacer es buscar el objetivo central y después dividirlo en actividades, lamentablemente nuestra visión ha pasado de la finalidad o misión para situarse tan solo en términos de la estructura. Al analizar el problema a través de objetivos, se puede obtener sus valores para el sistema total; en cambio, si se define el problema a través de departamentos (estructura) no es posible estimar su valor.

Por su parte Ackoff (1995) señala, que no sólo el diagnosticar el problema puede llegar a la solución del mismo, sino el permitir e incentivar a los elementos que forman parte del sistema para que se relacionen entre sí para aportar ideas y conocimientos. El mismo autor señala el momento de plantear, diseñar y administrar un sistema (proyecto) tratando de tener como punto de partida, el ideal es decir, a dónde se quiere llegar para no sentirnos limitados con los problemas que se presentan alrededor. Así mismo, hallar la satisfacción y eficiencia en cada parte del sistema para encontrar el agrado en los propósitos y necesidades del mismo, ésto, se puede lograr promoviendo la participación de los componentes del sistema e incentivando la creatividad y el intercambio de ideas.

Un objetivo es algo que importa lo suficiente como para realizar un esfuerzo para alcanzarlo. Un objetivo no es lo mismo que un deseo, por lo que para éste no se tiene intención alguna de intentar conseguirlo. Bajo el proceso de Planeación Estratégica, Ackoff (2008) determina los objetivos dentro de la fase de Planificación de Fines. En la Lámina 1, se presentan las cinco fases de la planeación estratégica y el lugar donde se ubican los objetivos.

Lámina 1. Las cincos fases de planeación

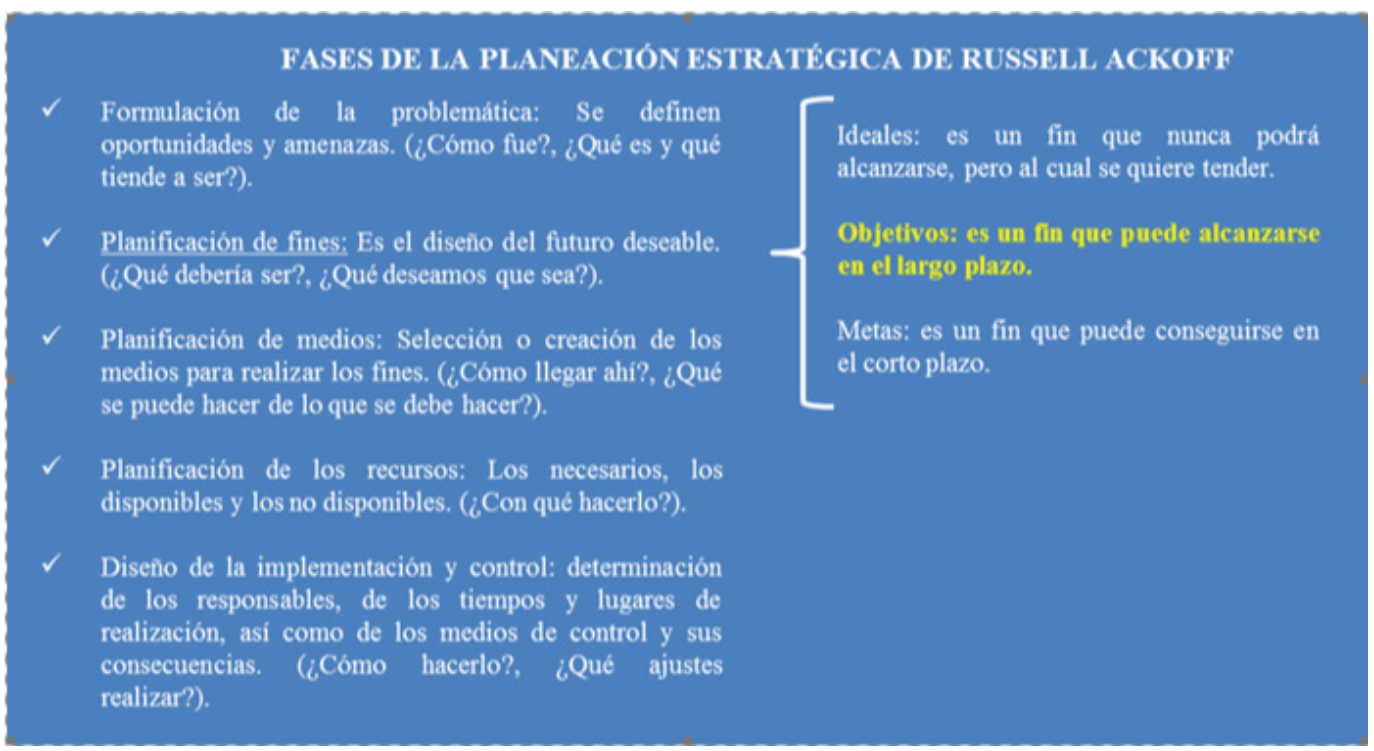

Fuente: Propia, basada en el proceso de Planeación Estratégica de Ackoff (2008) 
En sí, el proyecto para Haynes y Tejer (2007) debe ser el logro consecutivo de objetivos, que son el medio de dirección. El adecuado seguimiento y control disminuye las discrepancias entre lo que se espera y lo que en realidad se logra. Todo proyecto se enmarca en tres condicionantes; el alcance, el costo y el tiempo. Se deben cumplir con los objetivos del proyecto, acorde a lo presupuestado y realizarse en los tiempos preestablecidos. Como estos objetivos se componen de una serie de metas cuantificables, se está definiendo también cuáles son los entregables conforme a estas tres condicionantes. Un objetivo tiene cuatro componentes, el primero es el atributo o dimensión especifica que lo define; el segundo, la escala de medida; la norma o umbral es el tercer componente y el horizonte temporal constituyen el cuarto componente. En general, los objetivos de un proyecto se clasifican de la manera en que se muestra en la Tabla 1.
El objetivo primario o general, debe responder explícitamente a la pregunta principal. Puede haber uno o más objetivos secundarios específicos, que correspondan a aspectos parciales de la pregunta o a otros resultados derivados que también vayan a obtenerse en el proyecto. Los objetivos deben cumplir con las siguientes características:

a) Convenientes: Apoyan los propósitos de la misión.

b) Factibles: Deben ser posibles y aceptables.

c) Explícitos: Deben expresarse de forma clara y resumida, con lenguaje sencillo y evitando ambigüedades.

d) Medibles: Deben expresarse de tal modo que permitan ser medidos en sus cualidades o características propias.

e) Alcanzable: Sólo con el establecimiento de metas es posible lograrlo.

f) Obligatorios: Una vez que se logre el consenso debe existir el compromiso por lograr el objetivo.

Tabla 1. Clasificación de objetivos

\begin{tabular}{|c|l|}
\hline OBJETIVOS & \multicolumn{1}{|c|}{ TIPO } \\
\hline Generales & $\begin{array}{l}\text { Corresponden a las finalidades genéricas de un } \\
\text { proyecto o entidad. }\end{array}$ \\
\hline Especificos & $\begin{array}{l}\text { Se derivan de los objetivos generales. Señalan los } \\
\text { efectos específicos que se quieren conseguir. }\end{array}$ \\
\hline Operativos (metas) & $\begin{array}{l}\text { Concretan los objetivos especificos. Son } \\
\text { medibles y cuantificables. }\end{array}$ \\
\hline & Fuente: Propia
\end{tabular}

Moody (2002) considera que los proyectos son significan asignaciones presupuestales programadas. limitados en el tiempo, requiere que las fechas Un problema recurrente es la identificación y de conclusión de los objetivos se cumplan. Estas programación de estos objetivos parciales. En la Tabla acciones no pueden tratarse con ligereza, ya que estos 2 , se presenta la identificación de objetivos parciales objetivos además de inferir tiempos de ejecución, como funciones de respuestas esperadas.

Tabla 2. Objetivos Parciales de un Proyecto

\begin{tabular}{|c|l|}
\hline Objetivo - Restricción & No hay objetivo sin restricción. \\
\hline Objetivo - Preferencias & $\begin{array}{l}\text { Se manifiestan preferencias frente a las } \\
\text { alternativas. }\end{array}$ \\
\hline Objetivo - Poder & Se asignan los objetivos. \\
\hline Objetivo - Comunicación & $\begin{array}{l}\text { La comunicación de los objetivos refuerza } \\
\text { la coalición. }\end{array}$ \\
\hline Objetivo - Participantes & $\begin{array}{l}\text { Tos objetivos se sujetas a las necesidades } \\
\text { a cumplir con el proyecto. }\end{array}$ \\
\hline
\end{tabular}

Fuente: Propia 
Para comprobar si se están logrando los objetivos parciales, que pueden ser vistos como productos entregables durante la ejecución del proyecto, se requiere verificar que esos resultados en conjunto produzcan el efecto deseado. De esta manera el objetivo parcial contribuye de manera definitiva al logro del objetivo general. La secuencia lógica del proceso se presenta en la Lámina 2.

Lámina 2. Secuencia lógica del proceso de ejecución de un proyecto

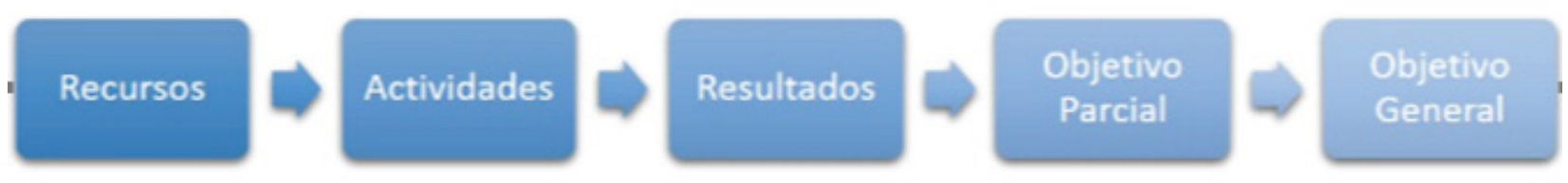

Fuente: Propia

Una manera para establecer las situaciones y Como se puede ver en la tabla anterior, aparecen en la condiciones en que se han de lograr los objetivos, es primera columna las "Intervenciones" que no es otra por medio de la matriz de planificación de proyecto. La cosa, que un resumen descriptivo de cada uno de los Tabla 3, muestra tal referencia. elementos del proceso. La siguiente columna se refiere a

Tabla 3. Matriz de Planificación de un Proyecto

\begin{tabular}{|l|l|l|l|l|}
\hline & $\begin{array}{c}\text { Logica de la } \\
\text { intervención }\end{array}$ & $\begin{array}{c}\text { Indicadores } \\
\text { objetivamente } \\
\text { cuantificables }\end{array}$ & $\begin{array}{c}\text { Fucntes de } \\
\text { verificación }\end{array}$ & Supuestos \\
\hline Objetivo Gencral & & & \\
\hline Objetivo Parcial & & \\
\hline Objetivo Parcial ....(n) & & & \\
\hline Resultados & & \\
\hline Actividades & &
\end{tabular}

Fuente: Propia

"Indicadores objetivamente verificables" donde se trata de precisar de manera inequívoca el grado del éxito que se espera, y que sean además comprobables. "Fuentes de verificación", en la tercera columna de la matriz, se presenta el procedimiento que se pondrá en marcha para comprobar de manera práctica el cumplimiento de los indicadores. Por último se presenta la columna de "Supuestos", factores externos o hipótesis, que son el conjunto de elementos de entorno que son importantes para que el proyecto tenga las condiciones para asegurar su éxito. Los supuestos también pueden ser vistos como los riesgos previsibles.

\section{Problemática}

Generalmente cuando un proyecto fracasa se le asocia al factor económico que a fin de cuentas es el efecto de diversas causas. Cualquier fracaso significa destinar recursos propios o externos para realizar acciones que no logran beneficios.

Para Tierouf (1997) la falla en los proyectos arrastra incumplimiento de objetivos totales y parciales, con ello, la insatisfacción de clientes y proveedores así mismo los participantes de éste sólo lograrán desánimo, desgaste y en consecuencia, el deterioro en la propia organización. Los clientes pierden mercado, reputación y sólo ganan un impacto negativo en sus finanzas. Si bien es cierto, existen muchas razones del porqué un proyecto puede fracasar, algunas más simples y otras más complejas, pero su número en verdad puede ser infinito. Las razones más comunes y frecuentes, sin que por esto se mantenga un grado de jerarquía son:

1- Falta de Comunicación: Si en la empresa no se toma el tiempo para comunicar lo que es el proyecto, es difícil que los involucrados sepan de qué se trata, de lo que se debe conseguir y de cómo llevarlo a cabo. La falta de identificación por parte de los involucrados es, en buena parte, su grado de compromiso e indiferencia. Sin la colaboración y compromiso de los interesados los proyectos difícilmente se logran.

2- Insipiente planificación: La planificación 
da principio con el alineamiento estratégico a la misión de la empresa, de ahí se derivan acciones tales como objetivos y metas que se integran en el proyecto.

3- $\quad$ Falta de objetivos: Las organizaciones como sistema de vida, deben estar orientadas al planteamiento de metas y objetivos para conseguir lo que se quiere. El no plantear objetivos, implica nulificar esfuerzos.

4- $\quad$ Falta de recursos: Tanto los materiales como los humanos son inadecuados o escasos.

5- Verificar resultados sólo hasta el final del proyecto: Analizar los resultados únicamente al final del proyecto, significa que las discrepancias, errores u omisiones nunca fueron atendidas.

6- Decisiones desafortunadas: La toma de decisiones carece de sustento informativo o de herramientas técnicas adecuadas.

7- Falta de control: Es común que la alta gerencia no sepa acerca del estado de avance del proyecto. La razón es que no se aplican las técnicas y herramientas adecuadas de seguimiento.

8- Inadecuada administración de riesgos: Todo proyecto se ve envuelto en una atmósfera de riesgos. Tanto los efectos positivos como los negativos, deben ser identificados para buscar medidas de mitigación.

La mala identificación o falta de los objetivos parciales, que es el tema del presente trabajo, es como ya se dijo, una de las principales causas del fracaso de los proyectos. Es necesario saber qué se quiere conseguir, cuándo y dónde. A continuación se presenta algunos de los errores más comunes al momento de formular tales objetivos:

*No considerar los resultados de proyectos ya realizados.

*No considerar el objetivo general, que a fin de cuentas es lo que le da sentido al proyecto.

* No identificar los objetivos con el responsable ejecutivo. Asignar varios responsables para el mismo objetivo.

*No fijar sub-objetivos o fechas intermedias que permitan medir el progreso de cada objetivo.
* No admitir ideas externas ni permitir que los subordinados expresen su opinión.

\section{GANTT, Acción fallida}

Otra manera común de pretender identificar los objetivos parciales es recurrir al gráfico de barras horizontales realizado por Henry Laurence Gantt (1861-1919), que intenta establecer la duración de la agenda de un proyecto expresada en una lista de actividades (una por línea) donde la dimensión es el tiempo. La Tabla 4, da cuenta de lo anterior.

Tabla 4. Ejemplo de Modelo Gantt

\begin{tabular}{|c|c|c|c|c|c|c|c|c|}
\hline Actividad & Enero & Febrero & Marzo & Abril & Mayo & Junio & Julio & TOTAL \\
\hline A & $\$ 200.00$ & $\$ 200.00$ & $\$ 200.00$ & $\$ 200.00$ & $\$ 200.00$ & & & $\$ 1,000.00$ \\
\hline B & & $\$ 510.00$ & $\$ 510.00$ & $\$ 510.00$ & & & & $\$ 1,530.00$ \\
\hline C & & & $\$ 400.00$ & $\$ 400.00$ & $\$ 400.00$ & $\$ 400.00$ & $\$ 400.00$ & $\$ 2,000.00$ \\
\hline Total por mes & $\$ 200.00$ & $\$ 710.00$ & $\$ 1,110.00$ & $\$ 1,110.00$ & $\$ 600.00$ & $\$ 400.00$ & $\$ 400.00$ & $\$ 4,530.00$ \\
\hline
\end{tabular}

Fuente: Propia

Como medio para establecer los objetivos parciales, el Gráfico de Gantt no ofrece las condiciones mínimas para el análisis. Kendall y Kendall, (1997) presentan algunas de las limitantes del Diagrama;

a) El diagrama de Gantt se revela muy eficaz en las etapas iniciales de la planificación, pero después se torna confuso y vago. b) Los proyectos son a menudo considerables más complejos como para poder ser expresados con eficacia a través de un diagrama de Gantt.

c) Los diagramas de Gantt representan sólo la parte de los apremios de los proyectos, ya que se centran sobre todo en el cronograma establecido (tiempo). 
d) No se presenta coordinación de actividades.

e) No existe una secuencia lógica de actividades.

f) No existe jerarquía de actividades.

g) No se muestran las relaciones de procedencia entre actividades.

h) No permite optimizar el desarrollo del programa.

i) No se identifica el impacto que significa el retraso de alguna actividad.

j) No se muestran las actividades críticas del proyecto.

Como se puede apreciar el Diagrama de Gantt no es el medio más apropiado para determinar los objetivos parciales de un proyecto.

Para validar los objetivos se debe analizar periódicamente el grado de avance, los resultados, alcanzados y el cumplimiento de la planificación prevista. Se debe contar con herramientas de análisis y toma de decisiones.

Camino más Largo

El método de la Ruta Crítica o de Camino más Largo es una herramienta de la administración y la gestión de proyectos, desarrollado en 1957 por los ingenieros R. Walter de la empresa Du Pont y por E. Nelly de la Rémington Rand, ambos introdujeron esta técnica para estimar los tiempos de duración de una serie de actividades de un proyecto, para determinar aquellas actividades que por su duración y secuencia se hacen críticas para la terminación del mismo. Lo que permite la planificación, la programación y el control de proyectos Wilson (1983). La Oficina de Proyectos Especiales de la Marina de Guerra de los Estados Unidos, realizó una de sus primeras aplicaciones de la Ruta Crítica en el proyecto Polaris, para la construcción de misiles balísticos para ser lanzados desde un submarino.

El método de la Ruta Crítica o PERT (Project Evaluation and Review Techniques), se extiende y diversifica en un sinfín de aplicaciones. Por ejemplo, en una relación contractual, el cliente puede imponer la penalidad respecto al plazo de entrega del bien o servicio.

Montaño (2002) señala que la Ruta también puede establecer la reducción de los tiempos de ejecución al mínimo costo, la identificación de actividades críticas en cuanto a sus tiempos de ejecución, la programación del manejo de la mano de obra y el programa óptimo de adquisiciones.

Taha (1995) señala que recurriendo al PERT es posible establecer las actividades requeridas para cumplir cada uno de los objetivos parciales, y no sólo eso, también es posible definir de cada uno, la programación en cuanto a los requerimientos de; mano de obra, recursos económicos, requerimiento de insumos y materiales, todo en tiempos específicos.

La Ruta Crítica o Técnica del Camino más Largo, de acuerdo a Poggioli (1976), se utiliza en escenarios de gestión de proyectos, es eficaz en su planeación y administración y facilita el manejo contable de la incertidumbre. Es la representación del plan de un proyecto donde se describe la secuencia o interrelación de todos los componentes del proyecto, así como el análisis lógico. Permite identificar situaciones del proyecto donde el riesgo (falta de holguras) es crítico y por lo tanto requieren de mayor atención y seguimiento. Por otra parte, para Gould (2010), la misma técnica muestra las bondades de flexibilidad en el tiempo de las actividades. Como se comentó anteriormente, la programación de proyectos por el Camino más Corto consta de tres funciones básicas:

Planeación; Se inicia por la descomposición del proyecto en sus distintas actividades. La construcción de diagramas de las redes tiene la ventaja de estudiar los diferentes trabajos a nivel de detalle, permite plantear mejoras antes de que el proyecto se ponga en marcha. Programación; En esta fase se construye la representación gráfica de un diagrama de tiempo, donde se muestra los tiempos de iniciación y terminación para cada actividad, así como su relación y dependencia con otras actividades del proyecto. El programa identifica las actividades críticas en función del tiempo que requieren atención especial, si es que se quiere que el proyecto se termine oportunamente. En el resto de las actividades no críticas, se muestra la holgura que bien pueden utilizarse cuando las actividades se demoran, o cuando se deben usar eficientemente recursos limitados. Control; El seguimiento adecuado del progreso de las actividades permite la identificación de discrepancia entre lo realizado y lo programado, es el medio más adecuado para actualizarse y analizar, si es necesario determinar un nuevo programa para la parte restante del proyecto.

A continuación se presenta el procedimiento realizado 
para determinar los objetivos parciales a partir del Camino más Largo.

\section{Los Objetivos Parciales del Proyecto}

Como se ha comentado anteriormente, el objetivo parcial es el efecto de la suma de actividades, la obtención de los resultados permitirá la consecución del objetivo parcial, expresado en términos de la modificación de la situación anteriormente existente. En la Lámina 3, se retoman las ideas de Fuentes (2003) para la integración de conceptos y que, de manera práctica, se interconectan a través del objetivo parcial.

Lámina 3. Interconexión de conceptos para lograr objetivos parciales

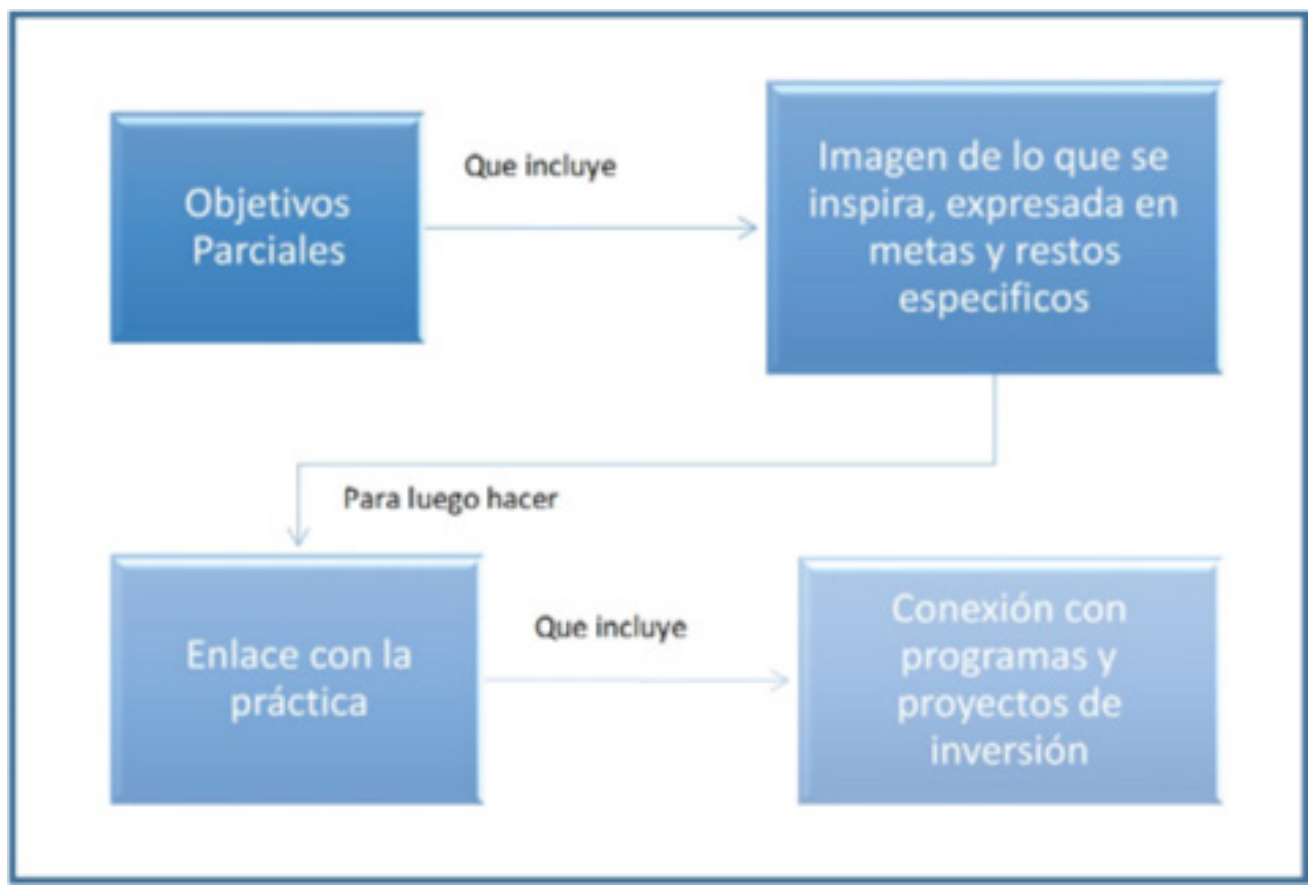

Fuente: Fuentes (2003).

La viabilidad de un proyecto se define como la medida en que los objetivos se cumplen. Evidentemente, la comprobación de la viabilidad final de un proyecto sólo puede determinarse cuando éste ha concluido su fase de ejecución, pero también se sabe que existen una serie de factores que condicionan su cumplimiento.

Una manera de reducir el riesgo en los proyectos de inversión es la generación de una serie de objetivos parciales. En el trabajo de planeación se desarrolla la llamada lista de comprobación, que por razones obvias no se aborda en este trabajo, pero sí las preguntas referentes a los objetivos, las cuales se presentan a continuación;

•¿El objetivo general es

suficientemente significativo como para justificar la realización del proyecto?

- ¿El objetivo permite cubrir la necesidad que se pretende resolver?

- ¿El objetivo se describe de forma operativa?

Por lo que hace al objetivo específico, éstas son algunas de las preguntas que se formulan en la lista de comprobación;

•¿Está bien definido el objetivo parcial?

- ¿El objetivo parcial contribuye a la obtención del objetivo general?

- ¿La conjunción de los objetivos parciales logra el objetivo general?

- ¿Los objetivos parciales son cuantificables y definidos en sus tiempos de ejecución?

Es evidente que una omisión en el establecimiento de los objetivos parciales pudiese frenar el desarrollo del proyecto.

Para un proyecto realizado es necesario establecer los objetivos parciales, dicho proyecto se encuentra en la etapa de factibilidad. En este estudio se reúne toda la información necesaria para evaluar un proyecto y los lineamientos generales para ponerlo en marcha. Los objetivos planteados para el proyecto se muestran a continuación:

1. Estudio de Mercado: Proceso de análisis de datos e información acerca de clientes, competidores, oferta y demanda de un producto o servicio. 
2. Análisis de disponibilidad de recursos y localización: Análisis de información para determinar la disponibilidad de los recursos con lo que cuenta la empresa y con ello determinar si se requieren más recursos para habilitarlos dentro de la región.

3. Estimación de costos y beneficios por alternativa: Estimación de gastos económicos y análisis de beneficios, con respecto a los gastos obtenidos, se identifican las mejores alternativas para manufactura.
4. Rentabilidad y selección de alternativas: Análisis de los recursos necesarios y los beneficios económicos para identificar las mejores alternativas, que permitan llevar a cabo la factibilidad del proyecto.

En la Tabla 5, se presentan las actividades del proyecto, las cuales se agrupan para conformar cada uno de los objetivos parciales. Cabe señalar que para pasar de un objetivo a otro es necesario que todas las actividades se tengan terminadas.

Tabla 5. Actividades para los objetivos parciales

\begin{tabular}{|c|l|l|}
\hline Objetivos Parciales & & \multicolumn{1}{|c|}{ Actividad } \\
\hline Estudio de Mercado & A & Oferta \\
\hline Demanda
\end{tabular}

Una vez determinadas las actividades, se realiza su costeo y se determinan los tiempos posibles de ejecución. Para realizar las estimaciones según Gallagher (1982) es necesario considerar la incertidumbre, de manera general se aplican tres estimadores de tiempos para cada actividad. El tiempo más probable $(\mathrm{m})$ : Es el tiempo que se requiere para terminar la actividad en condiciones normales. 2.- El tiempo pesimista (b): Es el tiempo máximo que se necesita para terminar la actividad si se presentan contratiempos o demoras considerables en el proyecto. 3.- El tiempo optimista (a): El tiempo mínimo que se requiere para terminar la actividad si todo ocurre en forma ideal. Con la aplicación de estos tres estimadores, puede calcularse un tiempo esperado
(Te) para la duración de una actividad. Para lo anterior, es necesaria la identificación de una distribución de probabilidades, en este caso y de manera práctica, se aplica la distribución Beta, ya que tiene las propiedades deseables de estar contenida de un intervalo finito y además puede ser simétrica o asimétrica, ésto dependiendo de la ubicación de modo de la relación con los estimaciones optimistas y pesimistas, Mendenhall, Scheaffer y Wackerly (2002).

Por lo que hace al tiempo esperado (Te) es tal que la probabilidad de que el tiempo real sea mayor o menor al 50\%, esto significa que el área bajo la curva es exactamente igual antes y después de este parámetro, 
escribe Navarro (2003). En la Lámina 4 se presentan los tres parámetros, la fórmula para calcular el tiempo esperado (Te) y los valores que se obtienen después de aplicar la fórmula.

Lámina 4. Tiempo Esperado (Te)

\begin{tabular}{|c|c|c|c|c|}
\hline Actividad & 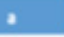 & $\mathrm{m}$ & $b$ & To \\
\hline A & 5.5 & 10.5 & 12.5 & 10 \\
\hline$B$ & 7.3 & 8.8 & 11.5 & 9 \\
\hline$c$ & 3 & 5 & 7 & 5 \\
\hline D & 4.4 & 10.5 & 13.6 & 10 \\
\hline E & 4.6 & 10.5 & 13.4 & 10 \\
\hline$F$ & 1 & 3 & 5 & 3 \\
\hline 6 & 11.5 & 14.5 & 20.5 & 15 \\
\hline H & 4 & 6 & 8 & 6 \\
\hline 1 & 5 & 7 & 9 & 7 \\
\hline 1 & 3 & 5 & 7 & 5 \\
\hline $\mathrm{K}$ & 2 & 4 & 6 & 4 \\
\hline$t$ & 2 & 3.5 & 8 & 4 \\
\hline $\mathbf{M}$ & 3.5 & 5 & 6.5 & 5 \\
\hline$N$ & 3 & 4.5 & 9 & 5 \\
\hline 0 & 3 & 5 & 7 & 5 \\
\hline$P$ & 4 & 5 & 6 & 5 \\
\hline $\mathbf{Q}$ & 3.5 & 4.5 & 8.5 & 5 \\
\hline R & 2 & 4 & 6 & 4 \\
\hline s & 2 & 3 & 4 & 3 \\
\hline$T$ & 1 & 2 & 3 & 2 \\
\hline$u$ & 0.8 & 2.2 & 2.4 & 2 \\
\hline
\end{tabular}

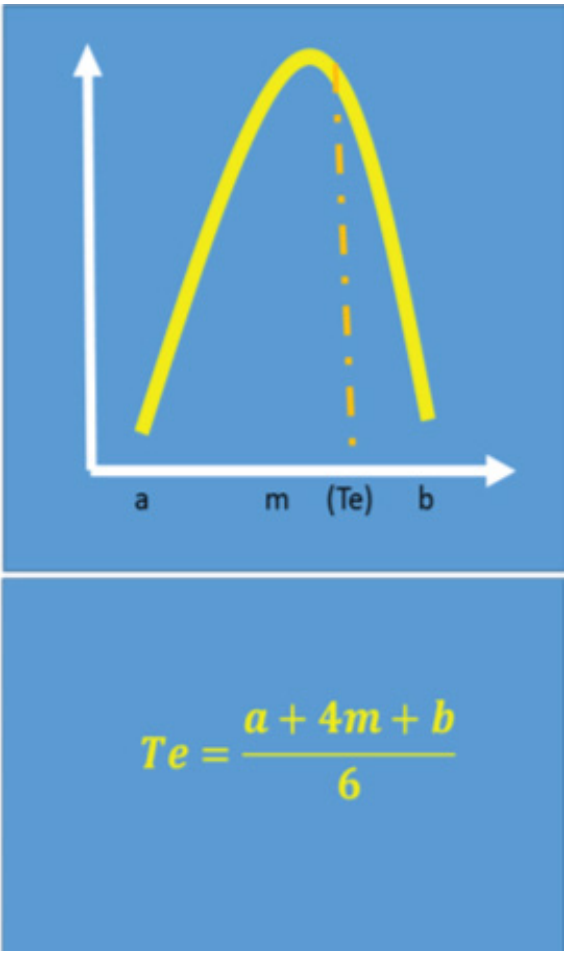

Fuente: Propia

La Tabla 6 presenta la información requerida para columna presenta la condicionante que la actividad elaborar la Ruta Crítica y la relación de actividades tiene para iniciar su proceso, lo anterior no significa que específicas, asociadas a su costo y tiempo esperado de sólo una actividad se esté desarrollando en un momento ejecución, que en este caso se expresa en días. La última dado Prawda (1982). 
Tabla 6. Actividades a realizar para el proyecto

\begin{tabular}{|c|c|c|c|c|}
\hline & $\overline{\text { Actividad }}$ & Costo Total & $\begin{array}{l}\text { Tiempo esperado } \\
\text { (Días) }\end{array}$ & $\begin{array}{l}\text { Actividad que } \\
\text { antecede }\end{array}$ \\
\hline $\mathrm{A}$ & Oferta & $\$ 75,000$ & 10 & - \\
\hline $\mathrm{B}$ & Demanda & $\$ 75,000$ & 09 & - \\
\hline $\bar{C}$ & $\begin{array}{l}\text { Proyección de } \\
\text { precios } \\
\text { Elasticidad } \\
\text { Tamaño }\end{array}$ & $\$ 50,000$ & 05 & $\mathrm{~A}, \mathrm{~B}$ \\
\hline $\bar{D}$ & $\begin{array}{l}\text { Disposición de } \\
\text { Mano de Obra }\end{array}$ & $\$ 25,000$ & 10 & $\mathrm{C}$ \\
\hline $\bar{E}$ & $\begin{array}{l}\text { Disposición } \\
\text { de Insumos y } \\
\text { Materiales } \\
\end{array}$ & $\$ 25,000$ & 10 & $\mathrm{C}$ \\
\hline $\bar{F}$ & $\begin{array}{l}\text { Determinación } \\
\text { de Proceso de } \\
\text { Producción }\end{array}$ & $\$ 30,000$ & 03 & $\mathrm{D}$ \\
\hline $\bar{G}$ & $\begin{array}{l}\text { Localización de la } \\
\text { Planta }\end{array}$ & $\$ 35,000$ & 15 & $\mathrm{D}$ \\
\hline $\mathrm{H}$ & $\begin{array}{l}\text { Requerimientos } \\
\text { de Maquinaria y } \\
\text { Equipo }\end{array}$ & $\$ 10,000$ & 06 & G \\
\hline I & Tamaño de Planta & $\$ 80,000$ & 07 & $\mathrm{H}$ \\
\hline $\bar{J}$ & $\begin{array}{l}\text { Costos de } \\
\text { Maquinaria }\end{array}$ & $\$ 5,000$ & 05 & $\mathrm{H}$ \\
\hline $\bar{K}$ & $\begin{array}{l}\text { Requerimientos } \\
\text { de Insumos y } \\
\text { Materiales } \\
\end{array}$ & $\$ 10,000$ & 04 & I \\
\hline$\overline{\mathrm{L}}$ & $\begin{array}{l}\text { Requerimientos de } \\
\text { Mano de Obra } \\
\end{array}$ & $\$ 10,000$ & 04 & I \\
\hline $\bar{M}$ & Costos de Insumos & $\$ 5,000$ & 05 & I \\
\hline $\mathrm{N}$ & $\begin{array}{l}\text { Costos de Mano de } \\
\text { Obra }\end{array}$ & $\$ 5,000$ & 05 & $\mathrm{~L}$ \\
\hline $\mathrm{O}$ & $\begin{array}{l}\text { Distribución de la } \\
\text { Planta }\end{array}$ & $\$ 12,000$ & 05 & $\mathrm{~K}$ \\
\hline$P$ & $\begin{array}{l}\text { Costos Beneficios } \\
\text { Alternativos } \\
\end{array}$ & $\$ 8,000$ & 05 & $\mathrm{O}$ \\
\hline $\bar{Q}$ & $\begin{array}{l}\text { Costos de } \\
\text { Distribución de } \\
\text { Planta } \\
\end{array}$ & $\$ 5,000$ & 05 & $\mathrm{~K}$ \\
\hline$\overline{\mathrm{R}}$ & $\begin{array}{l}\text { Análisi de Tamaño } \\
\text { Depreciación } \\
\text { Marginal }\end{array}$ & $\$ 16,000$ & 04 & $\mathrm{~J}, \mathrm{~N}, \mathrm{P}, \mathrm{Q}, \mathrm{M}$ \\
\hline $\mathrm{S}$ & Flujo de Caja & $\$ 15,000$ & 03 & $\mathrm{R}$ \\
\hline $\mathrm{T}$ & Rentabilidad & $\$ 17,000$ & 02 & $\mathrm{~S}$ \\
\hline $\mathrm{U}$ & Toma de Decisiones & $\$ 5,000$ & 02 & $\mathrm{~T}$ \\
\hline
\end{tabular}

Fuente: Propia

Para el desarrollo del Camino más Largo, como se comentó, se aplica la metodología desarrollada por Walter y Nelly. En un principio se establece el tiempo esperado (Te) de cada actividad cuyos parámetros se ajustan a la distribución de probabilidades llamada Beta. Posteriormente se calcula la Indicación Próxima y la
Alejada(IP, IA), por lo que se refiere a las Terminaciones Próximas y Alejadas (TP, TA) también se hace el cálculo. Martino (1970), propone que el resultado de la Ruta Crítica puede prestarse también por medio de una tabla, misma que se muestra a continuación: 
Tabla 7. Resultados de la corrida de la Ruta Crítica

\begin{tabular}{|c|c|c|c|c|c|c|c|c|c|}
\hline Actividad & a & m & b & Te & IP & TP & $\mathbf{L A}$ & $\mathbf{T A}$ & HT \\
\hline A & 5.5 & 10.5 & 12.5 & 10 & 0 & 10 & 0 & 10 & 0 \\
\hline B & 7.3 & 8.8 & 11.5 & 9 & 0 & 9 & 1 & 10 & 1 \\
\hline c & 3 & 5 & 7 & 5 & 10 & 10 & 10 & 15 & 0 \\
\hline D & 4.4 & 10.5 & 13.6 & 10 & 15 & 25 & 15 & 25 & 0 \\
\hline $\mathbf{E}$ & 4.6 & 10.5 & 13.4 & 10 & 15 & 25 & 15 & 25 & 0 \\
\hline $\mathbf{F}$ & 1 & 3 & 5 & 3 & 25 & 28 & 37 & 40 & 12 \\
\hline G & 11.5 & 14.5 & 20.5 & 15 & 25 & 40 & 25 & 40 & 0 \\
\hline и & 4 & 6 & 8 & 6 & 40 & 46 & 40 & 46 & 0 \\
\hline I & 5 & 7 & 9 & 7 & 46 & 53 & 46 & 53 & 0 \\
\hline $\mathbf{J}$ & 3 & 5 & 7 & 5 & 46 & 51 & 62 & 67 & 16 \\
\hline $\mathbf{K}$ & 2 & 4 & 6 & 4 & 57 & 61 & 57 & 62 & 0 \\
\hline $\mathbf{L}$ & 2 & 3.5 & 8 & 4 & 53 & 57 & 57 & 57 & 4 \\
\hline $\mathbf{M}$ & 3.5 & 5 & 6.5 & 5 & 53 & 58 & 62 & 67 & 9 \\
\hline $\mathbf{N}$ & 3 & 4.5 & 9 & 5 & 57 & 62 & 62 & 67 & 5 \\
\hline o & 3 & 5 & 7 & 5 & 57 & 62 & 57 & 62 & 0 \\
\hline $\mathbf{P}$ & 4 & 5 & 6 & 5 & 62 & 67 & 62 & 67 & 0 \\
\hline Q & 3.5 & 4.5 & 8.5 & 5 & 57 & 62 & 62 & 67 & 5 \\
\hline $\mathbf{R}$ & 2 & 4 & 6 & 4 & 67 & 71 & 67 & 71 & 0 \\
\hline s & 2 & 3 & 4 & 3 & 71 & 74 & 71 & 74 & 0 \\
\hline $\mathbf{T}$ & 1 & 2 & 3 & 2 & 74 & 76 & 74 & 76 & 0 \\
\hline $\mathbf{U}$ & 0.8 & 2.2 & 2.4 & 2 & 76 & 78 & 76 & 78 & 0 \\
\hline
\end{tabular}

Como se puede apreciar en la última columna (HT) de la tabla, se presentan las Holguras Totales por actividad. Para aquellas cuyo valor es cero, significa que cualquier atraso, en términos de tiempo, afecta de manera directa la fecha de terminación del proyecto. El resto de las actividades presentan holguras que no afectan directamente la conclusión del proyecto.

Schjetnan (2007), propone que una manera de representación del Camino más Largo es el medio gráfico, el que permite una mayor visualización del proceso, además de permitir identificar de manera más fácil las actividades a continuar de las que ahora se ejecutan y aquellas que resultan críticas para establecer medidas tendientes a tener un mayor control. En La Lámina 5, se muestra la red asociada a los objetivos antes citados.

La siguiente ilustración da a conocer los objetivos por los que está conformado todo el plan de actividades. Como se puede identificar, se establece un color por cada objetivo que se tiene que cumplir, indicando el seguimiento de cada actividad, para cumplir cada objetivo planteado. 
Lámina 5. Red asociada por objetivos

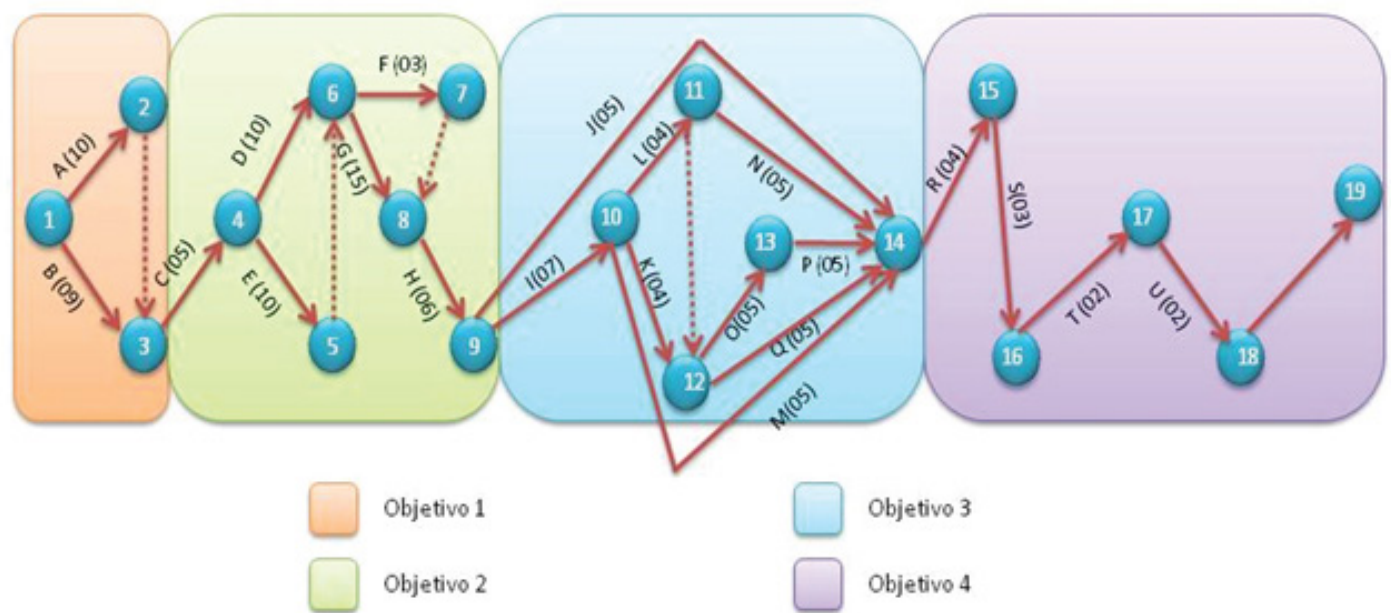

Fuente: Propia

En la Tabla 8, se presenta para el objetivo 1, el Estu- por dos actividades; oferta y demanda expresados en dio de Mercado que para este caso está conformado unidad de tiempo (días).

Tabla 8. Estudio de Mercado (Objetivo 1)

\begin{tabular}{|c|c|c|c|c|c|c|c|}
\hline A & Oferta & 10 & & & & & \\
\hline \multirow[t]{3}{*}{ B } & Demanda & 9 & & & & & \\
\hline & Días esperados por objetivo & & 10 & & & & \\
\hline & Semanas & 1 & 2 & 3 & 4 & 5 & 6 \\
\hline
\end{tabular}

Fuente: Propia

A continuación, se muestra la Tabla 9 correspondiente determina el proceso de producción (F) y en base a al objetivo 2. En este objetivo se analizan los éste, se establece la localización física de la planta requerimientos de los recursos $(C, D y E)$, estableciendo $(G)$ y finalmente los requerimientos de maquinaria y su calendarización de recursos. Posteriormente se equipo (H) de acuerdo a la proyección.

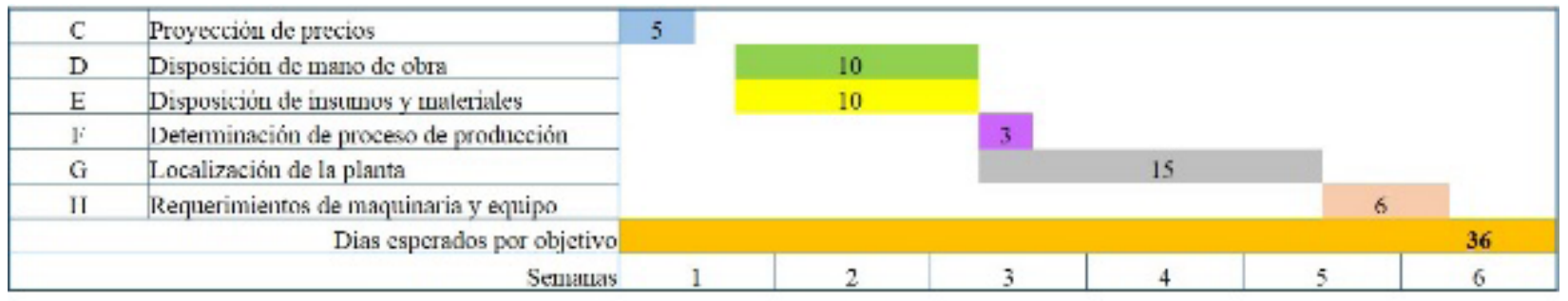

El objetivo 3 mostrado en la Tabla 10, permite identificar los tiempos requeridos para el diseño del tamaño de planta (I), los costos de la maquinaria $(J)$, requerimientos de insumos y materiales $(K)$, requerimientos de mano de obra $(L)$, costos de insumos (M) y mano de obra (N). También se identifica el tamaño de la planta, de acuerdo a la producción estimada. Es importante señalar que en este objetivo se incluyen las estimaciones de tiempo para determinar los costos y beneficios, y los costos referentes a la distribución de planta. 
Tabla 9. Análisis de disponibilidad de recursos (Objetivo 2)

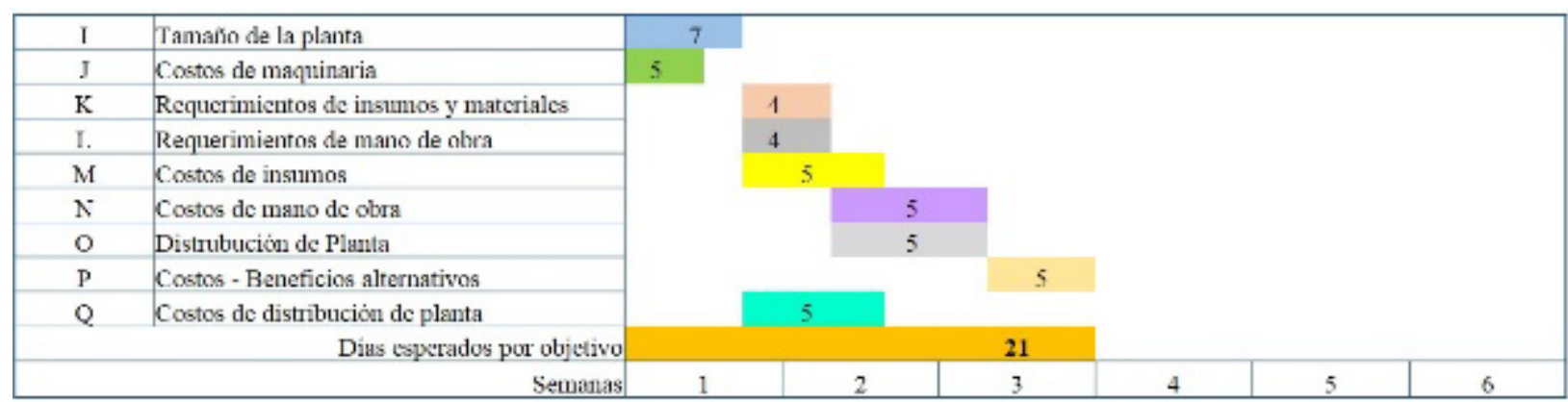

Fuente: Propia

El objetivo 4, da a conocer la rentabilidad y selección posible establecer los criterios para la adecuada toma de alternativas. Se identifica el análisis de tamaño, el de decisiones. flujo de caja y la rentabilidad, con esta información es

Tabla 11. Rentabilidad y selección de alternativas (Objetivo 4)

\begin{tabular}{|c|c|c|c|c|c|c|c|}
\hline $\mathrm{R}$ & Análisis de tamaño & 4 & & & & & \\
\hline $\mathrm{s}$ & Flujo de caja & & \multirow{3}{*}{4} & \multirow{4}{*}{1} & & & \\
\hline $\mathrm{T}$ & Rentabilidad & & & & & & \\
\hline $\mathrm{U}$ & Toma de decisiones & & & & & & \\
\hline & Dias esperados por objetivo & & & & & & \\
\hline & Semamas & 1 & 2 & 3 & 4 & 5 & 6 \\
\hline
\end{tabular}

Fuente: Propia

\section{Información derivada de los objetivos}

La información que se desprende de los objetivos parciales, no sólo se limita al inicio y final del tiempo del proyecto, también es posible derivar costos y su distribución programática. En cuanto a materias primas o insumos, es posible identificar sus requerimientos en el tiempo lo que facilita la programación en cuanto a pedido y licitaciones. En la Tabla 12, se presenta la distribución de los recursos económicos necesarios, que en este caso están programados por semanas.

Tabla 12. Costos por semana (Objetivo 3)

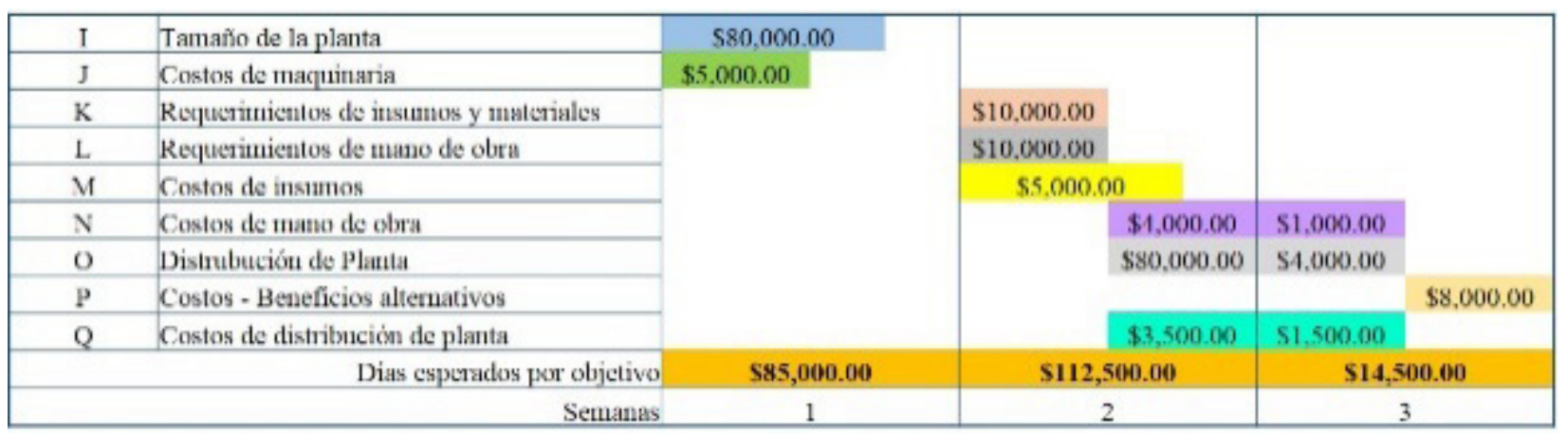

Fuente: Propia

Como ejemplo, para llevaruna adecuada administración de los recursos humanos se requiere de la formación y desarrollo del equipo de trabajo. Con los resultados obtenidos del Camino más Largo y su agrupación por objetivos parciales es posible conformar el equipo de trabajo; la secuencia es la siguiente:

1. Se identifican las competencias requeridas para efectuar el trabajo en los diferentes componentes del alcance.

2. Se identifican las personas (dentro o fuera) de la empresa que pudiesen realizar la actividad.

3. Se definen las responsabilidades de las personas y sus roles dentro del organigrama.

4. Se integran los grupos de trabajo para el proceso de desarrollo en equipo.

5. Con base a los objetivos parciales, se evalúa y orienta el trabajo de equipo.

6. Cuando se termine el proyecto se promueve la gestión del conocimiento.

De lo anterior y si es el caso, se puede recurrir a la 
Planeación Agregada que permite reducir tiempos muertos y eficientar el proceso. La Tabla 13, da cuenta de los requerimientos del costo de la mano de obra por semana para el mismo objetivo 3 .

Tabla 13. Requerimiento de mano de obra por actividad (Objetivo 3)

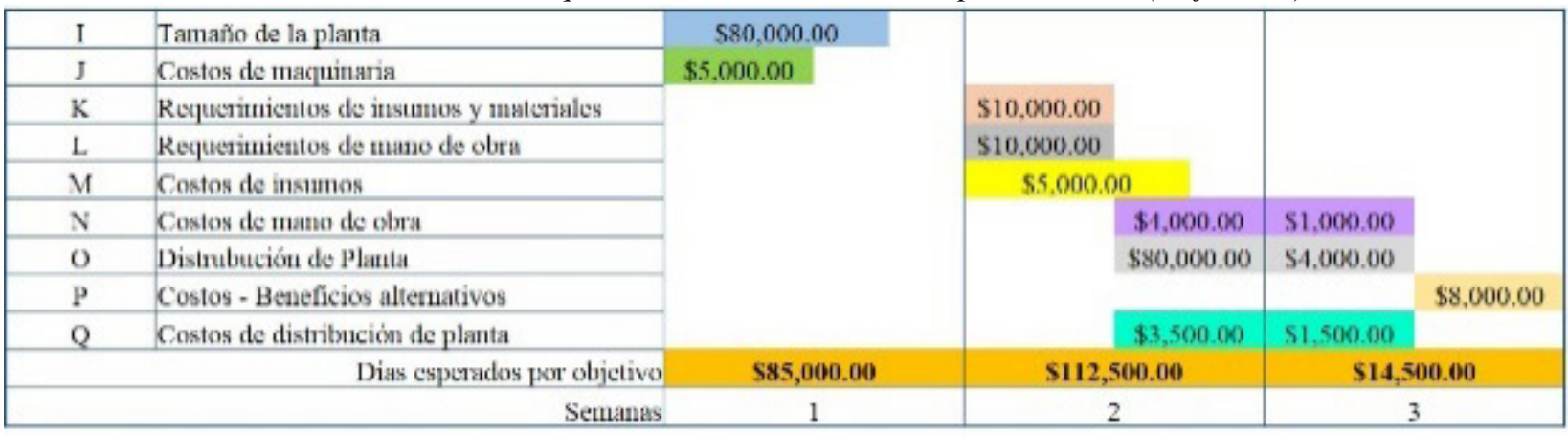

Fuente: Propia

\section{Conclusiones}

El establecer objetivos parciales en un proyecto de inversión sin un fundamento confiable, propicia entre otras cosas, un descontrol en la asignación y uso de los recursos, lo que en ocasiones impide o frena el desarrollo del propio proyecto. El no contar con los factores indispensables, que en este caso son las conjunciones de las actividades, no se garantiza el arribo a esos objetivos. La disfuncionalidad entre objetivos parciales tiene un impacto definitivo en el logro del objetivo principal, que a fin de cuentas es el que da sentido al proyecto.

Con la presente propuesta, es posible identificar los objetivos parciales, lo que evita que éstos sean sólo un producto de la imaginación. Con su identificación, los objetivos logran coherencia y se articulan en torno al objetivo general.

\section{Bibliografía}

Ackoff R. (2008), Planificación de la Empresa del Futuro, México, Limusa.

Ackoff R. (1995), Rediseñando el Futuro, México, Limusa Norega.

Antill, J (2001), Método de la ruta crítica y sus aplicaciones a la construcción, México, Limusa.

Churchman C.W. (1993), El Enfoque de Sistemas, México, Diana.

Fuentes A. (2003), Diseño de la Estrategia Competitiva, México, Planeación en Imágenes.

Gallagher, CH. (1982) Métodos cuantitativos para la toma de decisiones en administración, México, Mc Graw Hill Internacional.

Gómez, J. Coronel, A, Martínez L y Llorente (2000), Gestión de proyectos. Madrid, FC Editorial.
Gould, J. (2010), Investigación de Operaciones. México, Prentice Hall.

Haynes y Tejer. (2007), Administración de proyectos desde la idea hasta la implantación, México, Iberoamericana.

Kendall y Kendall, (1997). Análisis y Diseño de Sistemas. México, Prentice Hall.

Koontz, H.; Weihrich, H. (1994), Administration. Una perspectiva global, México, Mc Graw Hill,

Martino R. L. (1970), Administración y control de proyectos, México, Técnica.

Matus C, (1993), Estrategia y Plan, México, Siglo XXI. Mendenhall, Scheaffer y Wackerly. (2002), Estadística matemática con aplicaciones, México, Thomson.

Montaño, A. (2002), Iniciación al método del camino crítico. México, Trillas,

Moody, P.E, (2002), Toma de decisiones gerenciales, México. McGraw Hill.

Navarro H, (2003), Estadística aplicada, Madrid, Díaz de Santos.

Poggioli P. (1976), Aplicación Práctica del Método PERT. Barcelona, Técnicos Asociados. Barcelona.

Prawda, J. (1982), Métodos y modelos de investigación de operaciones. México, Limusa.

Robbins, S.P. y De Cenzo, A, (1994), Fundamentos de Administración. Conceptos y Aplicaciones, México, Prentice Hall Hispanoamericana.

Taha, H. (1995), Investigación de Operaciones. México, Alfa Omega.

Tierouf, R, (1997), Toma de decisiones por medio de investigación de operaciones. México, Limusa.

Schjetnan, M. (2007), Ruta crítica al alcance de todos, México, UNAM.

Wilson, R.J. (1983), Introducción a la Teoría de Grafos. Madrid: Alianza Universidad. 
Copyright (c) 2016 Rafael O. Sainz Zamora, Ana Rosa Cornejo Martínez, Mayra Morán Castrejón y Rosa Araceli Torres Castañeda

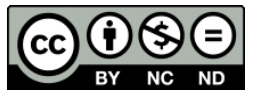

Este texto está protegido por una licencia Creative Commons 4.0

Usted es libre para Compartir (copiar y redistribuir el material en cualquier medio o formato), siempre que cumpla las condiciones de:

Atribución: Usted debe dar crédito a la obra original de manera adecuada, proporcionar un enlace a la licencia, e in dicar si se han realizado cambios. Puede hacerlo en cualquier forma razonable, pero no de forma tal que sugiera que tiene el apoyo del licenciante o lo recibe por el uso que hace de la obra.

No Comercial: Usted no puede hacer uso de la obra con propósitos comerciales

Sin Derivadas: Si remezcla, transforma o crea a partir de la obra, no podrá distribuir la obra modificada

$\underline{\text { Resumen de la licencia }}$ - Texto completo de la licencia 